\title{
NEW ASPECTS ON THE FRACTIONAL EULER-LAGRANGE EQUATION WITH NON-SINGULAR KERNELS
}

\author{
Norodin A. Rangaig \\ Department of Physics, Mindanao State University - Main Campus, 9700 Marawi City, Philippines \\ rangaig.norodin@msumain.edu.ph
}

Received: 1 April 2020; Accepted: 27 June 2020

\begin{abstract}
In this paper, we presented some notes in utilizing the fractional integral counterparts of the fractional derivatives with non-singular kernels on the action-like integral in Lagrangian mechanics. Considering a fractional integral, it may suggest that a dissipative term on the resulting fractional Euler-Lagrange equation can be obtained due to the imposed kernel. However, in the case of nonsingular kernel operators, different aspects of the fractional action-like integral were observed, and corresponding (fractionally-modified) Euler-Lagrange were derived, which imposes new insights on the dynamical system under the fractional regime.
\end{abstract}

MSC 2010: 26A33, 65R10, 93A30

Keywords: fractional derivative operator, non-singular kernel operator, fractional action-like integral, fractional Euler-Lagrange equation

\section{Introduction}

In the context of mathematical formulation on calculus, fractional calculus is one of the oldest, yet novel topics which has attracted many researchers for many decades. Recently, there have been many applications of this to various fields, and they are growing continuously. It is believed to be powerful in describing nonlinear phenomena since one can access a heterogeneity of the system through the given fractional order. To elaborate on the novelty of this fractional calculus topic, we refer to some of the following papers, and the references therein, to the readers [1-10]. In the past years, studying the dynamics of some physical system, through Lagrange's or Hamilton's equation by generalizing it to the fractional case, intrigued many scientists because of its capability in describing some fractional dimensions. Riewe [11] started these controversial fractional mechanics through the generalized fractional Lagrangian and Hamiltonian of the system for a nonconservative system. Later on, generalized variational problems and Euler-Lagrange equations were established by Agarwal [12], then followed by Tarasov and Zaslavsky [13] by presenting the fractional generalization of the nonholonomic constraints in the fractional derivative case. In 2013, Li and Luo [14] introduced the fractional generalized Hamiltonian 
mechanics. Some physical applications of fractional Lagrangian and fractional Euler-Lagrange equations have been studied for the dynamics of a physical system. In [15], they compared the classical and fractional dynamics of a coupled pendulum using a fractional derivative operator. Stachowiak and Okada [16] investigated the chaos in the double pendulum, and the forced double pendulum was presented in [17].

There is much-presented work regarding the formulation of the calculus of variations in the fractional scheme, in which remarkable results obtained since the resulting fractional Euler-Lagrange equation describes non-conservative terms such as friction [18]. Several discussions on the emerging of a dissipative term on the actionlike integrals were developed using Riemann-Liouville operators [18-20], and fixing the inconsistencies started by Riewe's formulation regarding fractional mechanics. In this work, we will consider the fractional derivatives with the non-singular kernel, namely, the Caputo-Fabrizio [21] and Atangana-Baleanu fractional derivatives [3]. To work the context of fractional calculus of variation within these frameworks, we will investigate if these fractional derivatives can capture the dissipative system according to the resulting fractional Euler-Lagrange equation. We can directly study the fractional action-integral using the fractional integral counterpart of the said derivatives. Within that scheme, we can directly apply the calculus of variation in fractional space. The main features of this work are:

- Fractional calculus of variations in the framework of non-singular fractional framework was studied.

- Corresponding fractional integrals of the derivative operators were used to formulate the action-like integral.

- Classical and fractionalized Lagrangian functions were considered in the presented framework to compare the resulting fractional Euler-Lagrange's equation.

- In the context of non-singular fractional operators, dissipative terms on the Euler-Lagrange equation were also obtained with a different form, as compared to the dissipative term that were previously reported in the framework of Caputo and Riemann-Liouville's operator and conformable derivative [22].

We organized the paper as follows: In Section 2, we started by giving some brief review about the definitions and properties of the nonsingular derivatives, which are all essential in the sequel of the study. We presented the fractional Euler-Lagrange equation in the framework of the two fractional derivatives and the classical and fractionalized Lagrangian of the system to obtain the corresponding Euler-Lagrange equation in Section 3.

\section{Mathematical preliminaries}

In this preliminary, we will present the definitions of the imposed fractional derivatives with the non-singular kernel, namely, the Caputo-Fabrizio and Atangana-Baleanu 
fractional derivatives. Firstly, we introduce the ancient definition of the Caputo fractional derivative and the Riemann-Liouville fractional integral.

Definition 1 Given a function $f \in H^{1}(a, b), a>b$ and $0<\alpha<1$, then the Caputo fractional derivative is defined as

$$
{ }^{C} D^{\alpha} f(t)=\frac{1}{\Gamma(1-\alpha)} \int_{0}^{t} \frac{f^{\prime}(s)}{(t-a)^{\alpha}} d s .
$$

From the definition above, one can define a fractional integral counterpart, known as the Riemann-Liouville fractional integral given by the definition:

Definition 2 Let $f \in H^{1}(a, b), a>b$ and $0<\alpha<1$, then the Riemann-Liouville fractional integral of order $\alpha$ defined as

$$
I_{t}^{\alpha} f(t)=\frac{1}{\Gamma(\alpha)} \int_{0}^{t} \frac{f(s)}{(t-a)^{1-\alpha}} d s .
$$

Note that these definitions are singular at $t=s$ which can account to a non-full memory kernel. To remedy these contexts, fractional derivatives were introduced and nonsingular kernels were imposed. According to equation (1), changing the kernel $(t-a)^{-\alpha}$ by the exponential function $\exp -\alpha(t-s) /(1-\alpha)$ and the normalization function $1 / \Gamma(1-\alpha)$ to $1 / \sqrt{(2 \pi(1-\alpha))}$, the new fractional derivative with a nonsingular kernel can be defined, and it is called the Caputo-Fabrizio fractional derivative [21], given by the definition:

Definition 3 Let $f \in H^{1}(a, b), a>b$ and $\alpha \in(0,1]$, then the Caputo-Fabrizio fractional derivative is defined as

$$
{ }^{C F} D^{\alpha} f(t)=\frac{1}{(1-\alpha)} \int_{0}^{t} f^{\prime}(s) \exp \left(-\frac{\alpha}{1-\alpha}(t-s)\right) d s .
$$

From the definition (3), the associated fractional integral can be derived and was shown by Losada and Neito [23]. Consider the fractional differential equation

$$
{ }^{C F} D^{\alpha} f(t)=g(t),
$$

then we have the definition:

Definition 4 Let $\alpha \in(0,1]$ be the fractional order of the Caputo-Fabrizio fractional derivative, then the associated fractional integral of a function $f \in H^{1}(a, b)$ is given by

$$
{ }^{C F} I^{\alpha} f(t)=(1-\alpha) f(t)+\alpha \int_{0}^{t} f(s) d s .
$$


Note that the fractional integral preserves the nature of the classical integral without any singularity, compared to the Riemann-Liouville fractional integral. Evidently, in studying physical systems, fractional derivatives with a nonsingular kernel show more accessible memory effects. On the other hand, Atangana and Baleanu [3] redefined the Caputo-Fabrizio fractional derivative by generalizing the exponential kernel to a one parameter generalized Mittag-Leffler function defined as

$$
E_{\alpha}\left(t^{\alpha}\right)=\sum_{k=0}^{\infty} \frac{t^{\alpha k}}{\Gamma(\alpha k+1)} .
$$

In which for $\alpha=1$, the function reduces to a series version of an exponential function.

Definition 5 Let $f \in H^{1}(a, b), a>b$ and $\alpha \in(0,1]$, then the Atangana-Baleanu fractional derivative is defined as

$$
{ }^{A B} D^{\alpha} f(t)=\frac{1}{(1-\alpha)} \int_{0}^{t} f^{\prime}(s) E_{\alpha}\left(-\frac{\alpha}{1-\alpha}(t-s)^{\alpha}\right) d s .
$$

This definition of the fractional derivative is more powerful since it generalizes the memory kernel imposed in the Caputo-Fabrizio fractional derivative $[5,6,8]$. However, both of these definitions contain a nonsingular kernel and give access to full memory, which is advantageous in studying the physical system in fractional space. Note that definition (5) follows a power-law kernel, which is the same as in the Caputo derivative, so it is also known as an Atangana-Baleanu fractional derivative in the Caputo sense. Now, following the same form of differential equation in (4), we can define the associated fractional integral.

Definition 6 Let $\alpha \in(0,1]$ be the fractional order of the Atangana-Baleanu fractional derivative in the Caputo sense, then the associated fractional integral of a function $f \in H^{1}(a, b)$ is given by

$$
{ }^{A B} I^{\alpha} f(t)=(1-\alpha) f(t)+\frac{\alpha}{\Gamma(\alpha)} \int_{0}^{t} f(s)(t-s)^{\alpha-1} d s .
$$

We have introduced the definitions of the nonsingular fractional derivatives together with their associated fractional integral counterparts. Additionally, the introduced fractional derivative operators are all linear operators and reduced to the classical calculus when $\alpha \rightarrow 1$. The important part of this work is the given associated fractional integrals. Both of the fractional integrals in (5) and (8) have the same first term, which inspired this work in studying the fractional action-like integral. According to [18], the fractional action-like integral can be reduced to the fractional Euler-Lagrange equation with a dissipative term when the definition of the Riemann-Liouville fractional integral was utilized. With this notion, we will investigate the fractional action-like integral and its consequential fractional Euler-Lagrange equation to check if the claimed dissipative term can exist in nonsingular fractional derivatives. 


\section{Fractional action-like integral and fractional Euler-Lagrange equation}

In this section we will investigate the fractional action-like integral in the framework of the nonsingular fractional derivative, using their fractional integral counterpart to formulate the Euler-Lagrange equation. With the help of calculus of variation, we will first define a generalized path coordinate $q(t)$ with the variational principle, and we parametrize the path coordinate as

$$
q(\beta, t)=q(0, t)+\beta \eta(t),
$$

such that $\eta\left(t_{a}\right)=\eta\left(t_{b}\right)=0$, and $q(0, t)=q(t)$.

Proposition 1 If $q$ is an extremizable path, then applying the fractional derivative operators (3) and (7) to the parametrized path coordinate (9), we have

$$
{ }^{*} D_{t}^{\alpha} q(\beta, t)={ }^{*} D_{t}^{\alpha} q(0, t)+\beta{ }^{*} D_{t}^{\alpha} \eta(t) .
$$

We also recall the definition of fundamental theorem of the calculus of variation, which is very important in the subsequent discussions of this paper.

Definition 7 For any arbitrary continuous function $\eta(t)$ up to its second derivative, and if

$$
\int_{t_{a}}^{t_{b}} G(\tau) \eta(\tau) d \tau=0
$$

then $G(\tau)$ must identically vanish in the interval $\left[t_{a}, t_{b}\right]$.

Now, we can formulate the fractional action-like integral in the variational problem in the subsequent definitions.

Definition 8 Suppose we have a continuous manifold $T$ and let the $L$ be a classical Lagrangian function $L=L(q(t), \dot{q}(t) ; t): \mathfrak{R}^{3 d} \rightarrow \mathfrak{R}, d \geq 1$. So, for any smooth path $q:\left[t_{a}, t_{b}\right] \rightarrow T$ with a fixed boundary condition. We define the the fractional action integral under the nonsingular kernel scheme as:

$$
{ }^{*} S^{\alpha}[q]={ }^{*} I^{\alpha}(L(q(t), \dot{q}(t) ; t)),
$$

such that the notation * (...) hold for Caputo-Fabrizio and Atangana-Baleanu fractional integral operators.

Definition 9 Let the fractional Lagrangian function be $L=L\left(q(t),{ }^{*} D^{\alpha} q(t) ; t\right)$ : $\mathfrak{R}^{3 d} \rightarrow \mathfrak{R}$, then the we can write the fractional action integral under nonsingular kernel scheme as with fractional Lagrangian function as

$$
{ }^{*} S^{\alpha}[q]={ }^{*} I^{\alpha}\left(L\left(q(t),{ }^{*} D^{\alpha} q(t) ; t\right)\right) .
$$


From these definitions, we have generalized the action-like integral in terms of fractional integrals. The Lagrangian function can interplay between fractional and classical regimes according to the variation principle in equations (9)-(10). The aforementioned definitions in this section do not give any physical implication yet, for the sake of the mathematical aspect, we will follow the given definitions. One certain physical implication when $\alpha \rightarrow 1$ is known. We will know the importance and possible implications of fractional order imposed on the system in the subsequent theorems.

Theorem 1 (Caputo-Fabrizio operator) If $q:\left[t_{a}, t_{b}\right] \rightarrow T$ is the extermizable path coordinate of the action-like integral with the classical Lagrangian function $L=$ $=L(q(t), \dot{q}(t) ; t): \mathfrak{R}^{3 d} \rightarrow \mathfrak{R}$, then

$$
{ }^{C F} S^{\alpha}[q]=(1-\alpha)(L(q(t), \dot{q}(t) ; t))+\alpha \int_{0}^{t}(L(q(s), \dot{q}(s) ; s)) d s .
$$

So, from the calculus of variation and extremizing ${ }^{C F} S^{\alpha}[q]$, the Euler-Lagrange equation is unaffected by fractionalizing the action-like integral. That is,

$$
\frac{\partial L}{\partial q}-\frac{d}{d t}\left(\frac{\partial L}{\partial \dot{q}}\right)=0
$$

(Proof): From the variational principle shown in equation (9), then the fractional action integral in (14) is extremum from $[0, t]$, that is,

$$
\frac{d}{d \beta} C F S^{\alpha}[q]=0 .
$$

So, in the framework of Caputo-Fabrizio fractional integral (5), we have

$$
(1-\alpha) \frac{d}{d \beta}(L(q(t), \dot{q}(t) ; t))+\alpha \int_{0}^{t} \frac{d}{d \beta}(L(q(s), \dot{q}(s) ; s)) d s=0 .
$$

Then, we rewrite the above expression as

$$
(1-\alpha) \underbrace{\int_{0}^{t} \frac{d}{d s}\left(\frac{d}{d \beta}(L(q(t), \dot{q}(t) ; t))\right) d s}_{A}+\underbrace{\alpha \int_{0}^{t} \frac{d}{d \beta}(L(q(s), \dot{q}(s) ; s)) d s}_{B}=0 .
$$

Evaluating the terms in equation (18), we start with $A$ and by applying integration by parts

$$
\begin{aligned}
A & =\int_{0}^{t} \frac{d}{d s}\left(\frac{d}{d \beta}(L(q(t), \dot{q}(t) ; t))\right) d s=\int_{0}^{t} \frac{d}{d s}\left(\frac{\partial L}{\partial q} \frac{\partial q}{\partial \beta}+\frac{\partial L}{\partial \dot{q}} \frac{\partial \dot{q}}{\partial \beta}\right) d s \\
& =\int_{0}^{t}\left[\frac{d}{d s}\left(\frac{\partial L}{\partial q}\right) \eta(s)+\frac{\partial L}{\partial q} \dot{\eta}(s)+\frac{d}{d s}\left(\frac{\partial L}{\partial \dot{q}}\right) \dot{\eta}(s)+\frac{\partial L}{\partial \dot{q}} \frac{d}{d s} \dot{\eta}(s)\right] .
\end{aligned}
$$


Simplifying further and applying repeated integration by parts, we get the final expression of the term

$$
A=\int_{0}^{t} \frac{d}{d s}\left[\frac{\partial L}{\partial q}-\frac{d}{d s}\left(\frac{\partial L}{\partial \dot{q}}\right)\right] \eta(s) d s+\int_{0}^{t}\left(\frac{\partial L}{\partial q}-\frac{d}{d s}\left(\frac{\partial L}{\partial \dot{q}}\right)\right) \frac{d}{d s} \eta(s) d s .
$$

Hence, by taking another integration by parts on the second term in the right-hand side of the equation (20), we can deduce that the term $A=0$. So, we are left with the term $B$ of equation (18), then the fractionalize action-like integral is proportional to the classical form and is weighted by the fractional order. With the help of the fundamental lemma of the calculus of variations, the term $B$ can be simplified. Therefore, the classical Euler-Lagrange equation is preserved under the fractionalization of an action-like integral. This completes the proof of theorem 1.

For the case of a fractionalized Lagrangian, we present the following Lemma of theorem 1 .

Lemma 1 If $q:\left[t_{a}, t_{b}\right] \rightarrow T$ is the extremizable path coordinate of the fractional action-like integral in the fractional space with fractional Lagrangian function $L_{\alpha}=$ $=L_{\alpha}\left(q(t),{ }^{C F} D q(t) ; t\right): \mathfrak{R}^{3 d} \rightarrow \mathfrak{R}$, then the Euler-Lagrange equation is fractionalized with the form

$$
\frac{\partial L}{\partial q}-\left[{ }^{C F} D_{t}^{\alpha}\left(\frac{\partial L}{\partial^{C F} D_{t}^{\alpha} q}\right)\right]=0
$$

Proof: We can express the fractional action-like integral with fractionalized Lagrangian function as

$$
{ }^{C F} S^{\alpha}[q]=(1-\alpha)\left(L_{\alpha}\left(q(t),{ }^{C F} D_{t}^{\alpha} q(t) ; t\right)\right)+\alpha \int_{0}^{t}\left(L_{\alpha}\left(q(s),{ }^{C F} D_{s}^{\alpha} q(s) ; s\right)\right) d s,
$$

and following the same steps made in proving theorem 1, we can get the result of the fractional Euler-Langrange equation because that were done fractionalizing the Lagrangian function.

From the two theorems presented above, we present the next corollary.

Corollary 1 Suppose that the path coordinate $q:\left[t_{a}, t_{b}\right] \rightarrow T$ is extremizable in both classical and fractional sense. Therefore,

$$
\frac{d}{d \beta}(L(q(t), \dot{q}(t) ; t))=\frac{d}{d \beta}\left(L_{\alpha}\left(q(t),{ }^{*} D_{t}^{\alpha} q(t) ; t\right)\right)=0 .
$$

The proof of corollary 1 can directly shown through simple theorems in fundamental calculus, and we leave the steps to the readers. It is evident that the claimed dissipative term in fractional Euler-Lagrange equation, claimed in [18-20, 22], is not present in the framework of the Caputo-Fabrizio operator for the classical Lagrangian. The nature of the Euler-Lagrange equation is preserved under this framework. In theorem 1, the physical sense of the Euler-Lagrange equation is independent 
of the fractional-order $\alpha$, considering a classical Lagrangian in fractional action-like integral. However, in the case of the fractionalizing Lagrangian function in a fractional action-like integral, we obtain a fractional Euler Lagrange equation (21). The physical significance of this formulation is not clear or may not have a physical meaning at all. To have a better view of formulation (21), we will consider the fundamental definition of the Lagrangian in a fractional sense, which is quadratic in ${ }^{C F} D_{t}^{\alpha} q(t)$ as

$$
L_{\alpha}=\frac{1}{2} m\left({ }^{C F} D_{t}^{\alpha} q(t)\right)^{2}-V(q),
$$

where the right-hand-side represents kinetic and potential energy. As we can see, this fractional Lagrangian function does not contain any frictional or dissipative terms. However, if we apply the formulation (21), the dissipative term will emerge. Now, substituting (24) to (21), we have the fractional equation of motion

$$
{ }^{C F} D_{t}^{\alpha}\left[{ }^{C F} D_{t}^{\alpha} q(t)\right]=\frac{1}{m} F(q(t)),
$$

where $F(q(t))=-\frac{d V(q)}{d q}$. Equation (25) can be rewritten in its equivalent form by applying the Laplace transform repeatedly, as

$$
m \ddot{q}(t)-\alpha^{2} F(q)=2 \alpha(1-\alpha) \frac{d F(q(t))}{d t} .
$$

Hence, the dissipative term is obtained for this formulation as predicted in a physical model $[17,24]$. Consequently, this is not the same kind of dissipative term as shown for Caputo's sense in $[19,20]$ and the conformable derivative sense [22]. A new definition can be presented as a consequence of formulation (21).

Definition 10 Let $q:\left[t_{a}, t_{b}\right] \rightarrow T$ be the extremizable path coordinate in the fractional scheme. Then, the fractional Euler-Lagrange equation for the fractional Lagrangian function $L_{\alpha}\left(q(t),{ }^{C F} D q(t) ; t\right)$ is defined as

$$
\frac{d}{d t} \frac{\partial L}{\partial \dot{q}}-\alpha^{2} \frac{\partial L}{\partial q}=2 \alpha(1-\alpha) \frac{d}{d t}\left(\frac{\partial L}{\partial q}\right)+(1-\alpha)^{2} \frac{d^{2}}{d t^{2}}\left(\frac{\partial L}{\partial q}\right),
$$

which is equivalent to the fractionalized Euler-Lagrange equation in (21).

Remark 1 Definition (10) is the fractional Euler-Lagrange equation in the framework of Caputo-Fabrizio operator, and the dissipative term is given on the right handside of the equation (27). The classical Euler-Lagrange equation is recovered when $\alpha \rightarrow 1$.

Remark 2 The dissipative term we obtain in (27) is called intrinsic dissipation since it can emerge when taking the equation of motion of a system from the Euler-Lagrange equation. A system dissipating its energy in the environment is the best description of this formulation. 
Let us now consider the Atanga-Baleanu operator (7)-(8). We start at the fractional action-like integral with classical Lagrangian function and derive the corresponding Euler-Lagrange equation.

Theorem 2 (Atangana-Baleanu operator) If $q:\left[t_{a}, t_{b}\right] \rightarrow T$ is the extremizable path coordinate of the action-like integral with the classical Lagrangian function $L=L(q(t), \dot{q}(t) ; t): \mathfrak{R}^{3 d} \rightarrow \mathfrak{R}$ in the Atangana-Baleanu framework, then

$$
{ }^{A B} S^{\alpha}[q]=(1-\alpha)(L(q(t), \dot{q}(t) ; t))+\frac{\alpha}{\Gamma(\alpha)} \int_{0}^{t}(L(q(s), \dot{q}(s) ; s))(t-s)^{\alpha-1} d s,
$$

such that the Euler-Lagrange equation takes the form

$$
\frac{\partial L}{\partial q}-\frac{d}{d t}\left(\frac{\partial L}{\partial \dot{q}}\right)=\frac{1-\alpha}{t-\tau} \frac{\partial L}{\partial \dot{q}}
$$

Proof: From the given fractional-action like integral in the Atangana-Baleanu sense and considering the fact that the path $q(t)$ is extremizable, the action is extremum if $\frac{d^{A B} S^{\alpha}[q]}{d \beta}=0$. By corollary 1 , the first term of (28) vanishes, and by variational principle,

$$
\int_{0}^{t} \frac{d}{d \beta}(L(q(s), \dot{q}(s) ; s))(t-s)^{\alpha-1} d s=0 .
$$

This can be simplified into

$$
\int_{0}^{t}\left(\left[\frac{\partial L}{\partial q}-\frac{d}{d t}\left(\frac{\partial L}{\partial \dot{q}}\right)\right](t-s)^{\alpha-1}+(\alpha-1)(t-s)^{\alpha-2}\left(\frac{\partial L}{\partial \dot{q}}\right)\right) \eta(s) d s=0,
$$

by the definition of a fundamental theorem in variational calculus. Therefore, we have

$$
\frac{\partial L}{\partial q}-\frac{d}{d t}\left(\frac{\partial L}{\partial \dot{q}}\right)=\frac{(1-\alpha)}{t-\tau} \frac{\partial L}{\partial \dot{q}}
$$

The Euler-Lagrange equation, with the classical Lagrangian, which contains a dissipative term as shown on the right-hand-side of equation (32) under the framework of the Atangana-Baleanu operator. This is significantly different from the obtain Euler-Lagrange equation under the framework of the Caputo-Fabrizio operator.

Definition 11 The equation (32) represents a classical Euler-Lagrange equation with a weighted generalized dessipative force

$$
Q_{\alpha}=\frac{(1-\alpha)}{t-\tau} \frac{\partial L}{\partial \dot{q}}
$$


Definition 12 Consider a generalized extremizable path coordinate $q:\left[t_{a}, t_{b}\right] \rightarrow T$ and a classical Lagrangian function $L(q(t), \dot{q}(t) ; t)$, then the classical Euler-Lagrange equation under the Atangana-Baleanu operator is

$$
\frac{\partial L}{\partial q}-\frac{d}{d t}\left(\frac{\partial L}{\partial \dot{q}}\right)=Q_{\alpha},
$$

such that $Q_{\alpha} \rightarrow 0$ when $\alpha \rightarrow 1$.

Comparing the two classical Euler-Lagrange equations between the framework of Caputo-Fabrizio (15) and Atangana-Baleanu (34), physical implications are directly observed. Hence, the Atangana-Baleanu operator gives more memory effect and direct physical implications than the Caputo-Fabrizio operator does. We present the Lemma below for the fractional Lagrangian function onto the fractional action-like integral under the framework of the Atanga-Baleanu operator.

Lemma 2 If $q:\left[t_{a}, t_{b}\right] \rightarrow T$ is the extermizable path coordinate of the fractional action-like integral in the framework of Atangana-Baleanu operator with fractional Lagrangian function $L_{\alpha}=L_{\alpha}\left(q(t),{ }^{A B} D^{\alpha} q(t) ; t\right): \mathfrak{R}^{3 d} \rightarrow \mathfrak{R}$, then the fractional Euler-Lagrange equation has the form

$$
\frac{\partial L}{\partial q}-\frac{1}{(t-\tau)^{\alpha-1}}\left[{ }^{A B} D_{t}^{\alpha}\left((t-\tau)^{\alpha-1} \frac{\partial L}{\partial^{A B} D_{t}^{\alpha} q}\right)\right]=0 .
$$

Proof: The proof of Lemma 2 is straightforward by evaluating the integral

$$
\int_{0}^{t}\left(\frac{\partial L}{\partial q} \eta(s)+\frac{\partial L}{\partial^{A B} D^{\alpha} q}{ }^{A B} D^{\alpha} \eta(s)\right)(t-s)^{\alpha-1} d s=0,
$$

and applying the integration by parts and the definition of fundamental theorem in calculus of variation.

Different types of Euler-Lagrange equations were derived from the framework of fractional operators. In a dynamic system, one can establish both the classical and fractionalize Lagrangian, depending on the system. Utilizing the fractional actionallike integral, we can then derive the Euler-Lagrange equation. However, a glimpse of physical implications can initially be established, but the real-world significance can point out whether these formulations are physically applicable when an experiment or direct observation were done. Additionally, even the fractionalized Lagrangian function can imply a fractional Euler-Lagrange equation, it can still be expressed as a classical Euler-Lagrange equation, so that the dissipative term can be extracted directly.

\section{Conclusion}

We have presented the new aspects of the Euler-Lagrange equation obtained from the fractional action-like integral under the framework of Caputo-Fabrizio and 
Atangana-Baleanu operators. The nature of the classical Euler-Lagrange equation is preserved under the Caputo-Fabrizio operator due to the limited memory kernel, compared to the framework of Atagana-Baleanu operator. The concept of additional dissipative term on the Euler-Lagrange equation was obtained under the Atagana-Baleanu operator. The nature of the Lagrangian of the system under this study is compared to a classical and fractionalized type in which the two operators give unique results based on the considered Lagrangian function. More importantly, the memory function in both operators is evidently essential in studying physical mechanics under fractional dimensions. Additionally, the initial findings of this paper are the inclusion of the dissipative term as shown in the obtained fractionally-modified Euler-Lagrange equation which is far different to the ones obtained using the Caputo operator, that contains a singular kernel function [13,18-20,25], and for the conformable operator [22]. Applications of the concepts found in this paper are an essential extension of this current work, such as the works done in $[26,27]$, the invariant conditions, Noether's theorem, and the DuBois-Reymond condition. Lastly, the continuation of this work will focus on finding the energy function, which can describe the origin of the found dissipative term on the fractionally-modified Euler-Lagrange equation and to further extend with their physical implications.

\section{Acknowledgements}

The author is highly thankful for the support imparted by the Department of Physics, Mindanao State University-Main Campus and to the Department of Science and Technology-Science Education Institute of the Philippines for the financial support throughout the past years. Special thanks to his wife whose support and love are unfathomable.

\section{References}

[1] Atangana, A., \& Alqahtani, R.T. (2016). Numerical approximation of the space-time Caputo-Fabrizio fractional derivative and application to groundwater pollution equation. Advances in Difference Equations, 2016(1), 1-13.

[2] Dokuyucu, M.A., Celik, E., Bulut, H., \& Baskonus, H.M. (2018). Cancer treatment model with the Caputo-Fabrizio fractional derivative. The European Physical Journal Plus, 133(3), 92.

[3] Atangana, A., \& Baleanu, D. (2016). New fractional derivatives with nonlocal and non-singular kernel: theory and application to heat transfer model. arXiv preprint arXiv:1602.03408.

[4] Algahtani, O.J.J. (2016). Comparing the Atangana-Baleanu and Caputo-Fabrizio derivative with fractional order: Allen Cahn model. Chaos, Solitons and Fractals, 89, 552-559.

[5] Atangana, A. (2018). Non validity of index law in fractional calculus: A fractional differential operator with Markovian and non-Markovian properties. Physica A: Statistical Mechanics and its Applications, 505, 688-706.

[6] Atangana, A., \& Gómez-Aguilar, J.F. (2018). Decolonisation of fractional calculus rules: breaking commutativity and associativity to capture more natural phenomena. The European Physical Journal Plus, 133(4), 166. 
[7] Qureshi, S., Rangaig, N.A., \& Baleanu, D. (2019). New numerical aspects of Caputo-Fabrizio fractional derivative operator. Mathematics, 7(4), 374.

[8] Atangana, A., \& Gómez-Aguilar, J.F. (2018). Decolonisation of fractional calculus rules: breaking commutativity and associativity to capture more natural phenomena. The European Physical Journal Plus, 133(4), 166.

[9] Rangaig, N.A., \& Convicto, V.C. (2019). On fractional modelling of dye removal using fractional derivative with non-singular kernel. Journal of King Saud University-Science, 31(4), 525-527.

[10] Rangaig, N. (2018). Finite difference approximation for Caputo-Fabrizio time fractional derivative on non-uniform mesh and some applications. Physics Journal, 1, 255-263.

[11] Riewe, F. (1997). Mechanics with fractional derivatives. Physical Review E, 55(3), 3581.

[12] Agrawal, O.P. (2010). Generalized variational problems and Euler-Lagrange equations. Computers $\mathcal{E}$ Mathematics with Applications, 59(5), 1852-1864.

[13] Tarasov, V.E., \& Zaslavsky, G.M. (2006). Nonholonomic constraints with fractional derivatives. Journal of Physics A: Mathematical and General, 39(31), 9797.

[14] Li, L., \& Luo, S.K. (2013). Fractional generalized Hamiltonian mechanics. Acta Mechanica, 224(8), 1757-1771.

[15] Baleanu, D., Jajarmi, A., \& Asad, J.H. (2019). Classical and fractional aspects of two coupled pendulums. ID: 209946116.

[16] Stachowiak, T., \& Okada, T. (2006). A numerical analysis of chaos in the double pendulum. Chaos, Solitons $\mathcal{E}$ Fractals, 29(2), 417-422.

[17] Rangaig, N.A., Pido, A.A.G., Pada-Dulpina, C.T. (2020). On the fractional-order dynamics of a double pendulum with a forcing constraint using the nonsingular fractional derivative approach. Journal of Applied Mathematics and Computational Mechanics, In Press.

[18] Almeida, R., Tavares, D., \& Torres, D.F. (2019). The Calculus of Variations. In The VariableOrder Fractional Calculus of Variations. Cham: Springer, 21-32.

[19] Almeida, R., \& Torres, D.F. (2011). Necessary and sufficient conditions for the fractional calculus of variations with Caputo derivatives. Communications in Nonlinear Science and Numerical Simulation, 16(3), 1490-1500.

[20] Barrios, M., \& Reyero, G. (2020). An Euler-Lagrange equation only depending on derivatives of Caputo for fractional variational problems with classical derivatives. Statistics, Optimization $\mathcal{E}$ Information Computing.

[21] Caputo, M., \& Fabrizio, M. (2015). A new definition of fractional derivative without singular kernel. Progress in Fractional Differentation and Application, 1(2), 1-13.

[22] Lazo, M.J., \& Torres, D.F. (2016). Variational calculus with conformable fractional derivatives. IEEE/CAA Journal of Automatica Sinica, 4(2), 340-352.

[23] Losada, J., \& Nieto, J.J. (2015). Properties of a new fractional derivative without singular kernel. Progress in Fractional Differentation and Application, 1(2), 87-92.

[24] Gómez-Aguilar, J.F., López-López, M.G., Alvarado-Martínez, V.M., Reyes-Reyes, J., \& Adam-Medina, M. (2016). Modeling diffusive transport with a fractional derivative without singular kernel. Physica A: Statistical Mechanics and its Applications, 447, 467-481.

[25] Baleanu, D., \& Muslih, S.I. (2019). Fractional Lagrangian and Hamiltonian mechanics with memory. Applications in Physics, 23.

[26] Mora, L.F.M. (2019). Explaining retrocausality phenomena in quantum mechanics using a modified variational principle. arXiv preprint arXiv:1907.09688.

[27] He, J.H., \& Ain, Q.T. (2020). New promises and future challenges of fractal calculus: from two-scale thermodynamics to fractal variational principle. Thermal Science, (00), 65-65. 